\title{
Bidens pilosa L. (Asteraceae) cultivated in Brazil on acute liver disease in dogs
}

[Bidens pilosa L. (Asteraceae) cultivada no Brasil na doença hepática aguda em cães]

\author{
A.V.C. Amaral ${ }^{1}$, L.M.L. Parente ${ }^{2}$, E.C. Conceição ${ }^{3}$, J.R. Paula ${ }^{3}$, L.C. Cunha ${ }^{3}$, \\ A.P.A. Costa ${ }^{4}$, L.S. Oliveira ${ }^{4}$, L.A.T. Benatti ${ }^{5}$, M.C.S. Fioravanti ${ }^{6}$ \\ ${ }^{1}$ Medicina Veterinária, Universidade Federal de Jataí - Jataí, GO \\ ${ }^{2}$ Medicina Veterinária, Universidade Salgado de Oliveira - Goiânia, GO \\ ${ }^{3}$ Faculdade de Farmácia, Universidade Federal de Goiás - Goiânia, GO \\ ${ }^{4}$ Aluna de Pós-Graduação em Ciência Animal, Universidade Federal de Goiás - Goiânia, GO \\ ${ }^{5}$ Universidade do Oeste Paulista - Presidente Prudente, SP \\ ${ }^{6}$ Medicina Veterinária, Universidade Federal de Goiás - Goiânia, GO
}

\begin{abstract}
Bidens pilosa $L$. is a medicinal plant popularly used for treatment of liver diseases. In this study, the dry extract of aerial parts of Bidens pilosa and Silymarin, a phytocomplex obtained from the Silybum marianum fruits and marketed as hepatoprotective, were tested in dogs experimentally acutely intoxicated with carbon tetrachloride. The liver activity was evaluated by hematological and biochemical profiles, and histological and ultrasound analyzes. It was observed that the lowest serum activities of ALT and serum concentrations of total bilirubin occurred in the groups treated with the dry extract of Bidens pilosa, while only decreased serum concentrations of total bilirubin occurred in the group treated with Silymarin. Best liver recovery was also observed for the dry extract of B. pilosa at a $400 \mathrm{mg} / \mathrm{Kg}$ dose by ultrasonography. This study showed that the dry extract of Bidens pilosa acted more efficiently in the treatment of acute toxic hepatitis induced in dogs than Silymarin.
\end{abstract}

Keywords: carbon tetrachloride, liver diseases, Bidens Pilosa

\section{RESUMO}

Bidens pilosa L. é uma planta medicinal utilizada popularmente para tratamento de doenças hepáticas. Neste trabalho, o extrato seco das partes aéreas da Bidens pilosa e a silimarina, um fitocomplexo obtido dos frutos da Silybum marianum e comercializado como hepatoprotetor, foram testados em cães intoxicados experimentalmente de forma aguda com tetracloreto de carbono. A atividade hepática foi avaliada por meio dos perfis hematológico e bioquímico, análises histológica e ultrassonográfica. Observou-se que, nos grupos tratados com o extrato seco da Bidens pilosa, ocorreram as menores atividades séricas da ALT e de concentrações séricas de bilirrubina total, enquanto no grupo tratado com silimarina, ocorreu apenas diminuição de concentrações séricas de bilirrubina total. Melhor recuperação hepática também foi verificada para o extrato seco de B. pilosa na dose de 400mg/kg por ultrassonografia. Este estudo evidenciou que o extrato seco da Bidens pilosa atuou de forma mais eficiente no tratamento da hepatite aguda tóxica induzida em cães do que a silimarina.

Palavras-chave: tetracloreto de carbono, doença hepática, picão

\section{INTRODUCTION}

Bidens pilosa $L$. belongs to the Asteraceae family originated from South America, is popularly known in Brazil as picão, picão preto, marcelado-campo and carrapicho de agulha and in the
USA as black-jack, beggar-ticks, cobbler's pegs and Spanish needle, and grows spontaneously in agricultural crops in all regions of Brazil. This is a cosmopolitan species and occurs in tropical and subtropical regions of the Americas and Asia (Lorenzi and Matos,

Recebido em 20 de março de 2019 
2008; Alonso, 2016). Flavonoids and polyacetylene compounds outstand among the chemical components of the aerial parts of $B$. pilosa. Phenylheptatriine, glycopyranosyl-OHtetradecene and glycopyranosyloxy-OHtridecene, quercetin 3-O-beta-D-glycoside, nicotinic acid, tannic acid, hydrocarbons with 28 30 carbons, among others have already been identified in this plant (Alonso, 2016; Silva et al., 2011). Some of the therapeutic activities associated with Bidens pilosa are antioxidative, immunomodulatory, anti-inflammatory and antiulcerogenic (Bartolome et al., 2013; Santos Filho et al., 2018). In Brazil, this species is included in the National List of Medicinal Plants of Interest to the Unified Health System - RENISUS, which aims to guide research and studies. There is even a specific monograph on Bidens pilosa prepared by the Ministry of Health (Brasil, 2015).

The aqueous extract of $B$. pilos $a$ has been used in case of liver injuries produced by acetaminophen and carbon tetrachloride in rats and decreased the injury areas significantly (Chin et al., 1996), also acting in cases of liver fibrosis in mice (Yuan et al., 2008). It has also showed antioxidant activity (Yang et al., 2006; Krishnaiah et al., 2011), what could contribute to the treatment of liver diseases.

Another plant used in liver diseases of different etiologies is Silybum marianum (L.) Gaertn (Asteraceae). A phytocomplex commercially known as Silymarin is extracted from the ripe and dry fruit of $S$. marianum. This phytocomplex features seven flavonolignans and polyphenols in its composition, and silybin is its main component (Alonso, 2016). In addition to its action in liver diseases, studies report that Silymarin shows antioxidant cytoprotective activity (Asghar and Masood, 2008) mediated by the transcription factor NFkB (Tsai et al., 2010), and anti-inflammatory and anticancer activity for suppressing the tumor necrosis factor (TNF) (Shulze-Osthoff and Haussinger, 2007; ElKamary et al., 2009). It is assumed that this phytocomplex is safe and effective in the improvement of symptoms in cases of acute hepatitis and in patients with alcoholic cirrhosis (Basu, 2003).

On the experimental evaluation of liver activity, carbon tetrachloride $\left(\mathrm{CCl}_{4}\right)$ has been used to induce liver injury in different experimental models, because this compound produces oxidative stress, with fatty acid and membrane phospholipid peroxidation, causing complete destruction of cell and intracellular membranes (Basu, 2003; Chen et al., 2005). The activity of a substance in the presence of liver injury induced by $\mathrm{CCI}_{4}$ may be evaluated by determining the serum activity of alanine aminotransferase (ALT), alkaline phosphatase (ALP) and gammaglutamyltransferase (GGT). In addition to these enzymes, other substances are correlated with the structural and functional integrity of the organ and may be analyzed, as the serum concentrations of albumin and urea (Chen et al., 2005). Thus, in this study, the liver activity of standardized dry extract of aerial parts of Bidens pilosa and Silymarin phytocomplex have been evaluated in dogs acutely intoxicated by oral administration of carbon tetrachloride.

\section{MATERIAL AND METHODS}

The experimental protocol was approved by the Animal Research Ethics Committee of the Universidade Federal de Goiás (process 164/2009). Aerial parts of Bidens pilosa L., in the form of plant drug, were acquired from Empresa Santa Efigênia (Goiânia, GO, Brazil). The morphological characteristics of this sample were analyzed considering the descriptions reported in the literature (Alonso, 2016; Lorenzi and Matos, 2008) and by comparison with a standard sample of Bidens pilosa L. deposited in the Herbarium of the UFG, under nr. 43835.

The aerial parts of $B$. pilosa were grounded in a knife mill (Wiley type), and the powder was submitted to percolation extraction using hydroalcoholic solution. The liquid extract was concentrated in a rotary evaporator at a temperature of $40^{\circ} \mathrm{C}$, up to about $40 \%$ of total solids, becoming the ethanolic extract of Bidens pilosa (EEB) and characterized regarding $\mathrm{pH}$, relative density, viscosity, alcohol content, solids content and total flavonoid content. The total flavonoid content was quantified by spectrophotometry and calculated as rutin, according to Rollim et al. (2005). The EEB was dried by nebulization/atomization, in spray drying, and was deposited in the Bank of Patents at the National Institute of Intellectual Property (BR10-2012 0076675). 
The Silymarin phytocomplex, in the form of dry extract, was provided by a magisterial pharmacy of Goiânia, GO, Brazil. This pharmacy also provided the fractionation and encapsulation of the Silymarin phytocomplex and EEB, according to the drug dose and weight of the dogs. Twentyfour adult mongrel male dogs from the Zoonosis Control Center of the city of Goiânia, with an average weight of $15 \pm 6.2 \mathrm{Kg}$ were used. At the end of the study, after confirming that the dogs were healthy, they were sent to foster homes. The animals were housed in individual pens and received balanced ration for adult dogs (Finotrato-VB rations) and water at ease, being monitored for a period of three months before the experiment.

For the administration of $\mathrm{CCl}_{4}$, the animals were anesthetized with propophol $(6 \mathrm{mg} / \mathrm{Kg} / \mathrm{EV})$ followed by placement of tracheal tube for orogastric probing. Carbon tetrachloride PA (99\%) was administered in a single dose of $2.5 \mathrm{~mL} / \mathrm{Kg}$. The dogs were distributed randomly into four groups $(n=6)$, which had the following treatments by oral administration, after the induction of liver injury: group I - Control (400mg/Kg starch), group II treated with EEB $(400 \mathrm{mg} / \mathrm{Kg})$, group III - treated with EEB $(100 \mathrm{mg} / \mathrm{Kg})$ and group IV treated with Silymarin $(30 \mathrm{mg} / \mathrm{Kg})$. Group I functioned as negative control and group IV as positive control. The treatments were performed once a day, at the same time, for a period of 21 days.

The animals were observed daily, throughout the experimental period, aiming at detecting episodes of vomiting, diarrhea, anorexia and any other change in the health conditions of the animals. For evaluating the enzymatic activity and other analytes, blood collections were conducted at the following times: before the intoxication, 24 hours after the intoxication induction and on days 2, 3, 5, 8, 11, 14, 19 and 21 after intoxication. The blood was collected by jugular venipuncture in a vacuum collection system (Vacutainer® - Becton Dickinson Ind. Cirúrgicas Ltda., Brazil).

The samples were processed in less than six hours as of the collection time. The counting of blood cells was conducted by an automatic method, using the BC-2800 vet device (Auto Hematology Analyzer, Mindray ${ }^{\circledR}$ Bio-Medical Electronics Co. Ltd., Shenzhen-Guangdong), adapted with a reading card proper to the canine species. For the differential counting of leukocyte, blood smears prepared with fresh blood and stained by the Rosenfeld technique were used.

For biochemical analyzes, standardized commercial reagents (Labtest® - Labtest Diagnóstica S.A., Lagoa Santa, MG, Brazil) were used, with kinetic, enzymatic and colorimetric methodologies, in a temperature of $37^{\circ} \mathrm{C}$, and the reading was conducted in a semiautomatic spectrophotometer (Analisador Bioquímico Bio-Plus $®)$. The reactions were performed according to the guidelines of the manufacturer. The evaluations included the determination of the serum activity of enzymes: alanine aminotransferase (ALT), aspartate aminotransferase (AST), alkaline phosphatase (ALP), gamma glutamyltransferase (GGT); quantification of total and direct bilirubin, total cholesterol, total protein, albumin, globulins, urea and creatinine.

Ultrasound monitoring was conducted in four evaluations throughout the experimental period, being: one before the intoxication, 48 hours after the intoxication, on the $7^{\text {th }}$ day and on the $21^{\text {st }}$ day. Abdominal images in real time were obtained with ultrasound equipment $\left(\mathrm{My} \mathrm{Lab}{ }^{\mathrm{TM}}\right.$ 30 Vet, The Esaote Group, Genoa, Italy) coupled to a multifrequency microconvex transducer (5.0 - $7.5 \mathrm{MHz}$ ). The longitudinal, dorsal and transverse section plans were used to the liver parenchyma scan. The liver was evaluated regarding size, echogenicity, echotexture and presence of biliary sludge, according to the Nyland et al. (2004) definitions.

The liver biopsy guided by ultrasonography was conducted when the animal was properly anaesthetized with isoflurane after induction with propophol $(6 \mathrm{mg} / \mathrm{Kg}, \mathrm{EV})$, using Tru-Cut ${ }^{\circledR}$ needle (CardinalHealth). Two fragments were removed (by examination) from the left medial liver lobe, positioning the needle at the right of the linea alba, below the last rib, with an average inclination of $35^{\circ}$ directed cranially. The liver fragments, collected by ultrasound-guided biopsy, were processed according to the histological routine and stained by hematoxylin and eosin (HE) in the Department of Pathology of the Escola de Veterinária e Zootecnia of the Universidade Federal de Goiás. The results were 
submitted to statistical treatment using the GraphPad InStat software (Version 3.05 for Windows). ANOVA and Tukey post-test were used for the analysis of parametric variables. The significance level was $\mathrm{P}<0.05$ (Sampaio, 2002).

\section{RESULTS}

The total flavonoids content of the EEB, calculated as rutin, was $366.8 \mathrm{mg} / 100 \mathrm{~g}$ of extract. This value is in accordance with literature data for this plant species (Brazil, 2015). In the first two days after the acute intoxication with $\mathrm{CCl}_{4}$ the animals of all groups showed clinical signs of vomiting and diarrhea. In the $100 \mathrm{mg}$ and $400 \mathrm{mg}$ EEB groups, vomiting ceased in the $3^{\text {rd }}$ and $4^{\text {th }}$ days after intoxication, respectively, while in the Silymarin and control groups vomiting remained until the $8^{\text {th }}$ day. Diarrhea episodes ceased in the $100 \mathrm{mg}$ and $400 \mathrm{mg}$ EEB groups on the $5^{\text {th }}$ day after intoxication and in the Silymarin and control groups it remained until the $18^{\text {th }}$ day. Anorexia was not observed in any of the groups evaluated. There was no death during the experimental stage.

No significant differences were observed $(\mathrm{P}>0.05) \quad$ concerning the hematological parameters between the experimental groups. The evaluation of the liver disease marker enzymes indicated that, 24 hours after intoxication of the dogs, there was significant increase in the serum activity of ALT, AST and ALP enzymes compared to the basal level. However, in the 72-hour time, there was significant decrease $(\mathrm{P}=0.001)$ of the serum ALT activity only in the groups treated with EEB $(100 \mathrm{mg} / \mathrm{Kg})$ and EEB $(400 \mathrm{mg} / \mathrm{Kg})$, compared to the negative control group (Table 1). Still regarding the ALT, significant increase was observed in the group treated with Silymarin $(\mathrm{P}<0.001)$, in the 24-hour time, compared to the negative control group (Table 1).

During the 11-day period after the intoxication, decrease $(\mathrm{P}=0.008)$ of the plasma concentration of total bilirubin was observed in the EEB groups $(400 \mathrm{mg} / \mathrm{Kg}$ and $100 \mathrm{mg} / \mathrm{Kg})$ and Silymarin compared to the group treated with starch, negative control (Table 2). In the same period, considering the direct bilirubin, a significant difference $(\mathrm{P}=0.02)$ was observed in the EEB $400 \mathrm{mg} / \mathrm{Kg}$ group compared to the negative control group (Table 2). Serum concentrations of urea and creatinine did not vary and remained within the physiological limits of the species. Regarding the size of the liver, hepatomegaly was observed in animals of all groups, 48 hours after intoxication. On the $7^{\text {th }}$ day, the animals treated with $400 \mathrm{mg}$ EEB already showed normal liver size. The animals of the other groups showed reduced liver size only on the $21^{\text {st }}$ day.

Forty-eight hours after intoxication, one of the animals of the EEB 100mg, 400mg EEB and control group showed diffuse hyperechogenicity of the liver parenchyma. On the $7^{\text {th }}$ day, this result was found in $50 \%$ of the $100 \mathrm{mg}$ EEB group and in $33.33 \%$ of the $400 \mathrm{mg}$ EEB and Silymarin groups. On the $21^{\text {st }}$ day, one of the animals of the 100mg EEB and 400mg EEB groups still showed diffuse hyperechogenicity. Regarding the echotexture, changes were seen on the $7^{\text {th }}$ day in $50 \%$ of the animals of the control group and in $33.33 \%$ of the other groups. On the $21^{\text {st }}$ day, this change was seen only in one dog of the Silymarin group.

In 48 hours, presence of biliary sludge was observed in $33.33 \%$ of the animals of the control and 400mg EEB groups, while in the $100 \mathrm{mg}$ EEB group this result was observed in $16.66 \%$. On the $7^{\text {th }}$ day, there was presence of biliary sludge in $33.33 \%$ in the control and 100mg EEB groups and $66.66 \%$ in the $400 \mathrm{mg}$ EEB group. On the $21^{\text {st }}$ day, this change was observed in $50 \%$ of the animals in the 400mg EEB group and in $16.66 \%$ in the control group. In the animals of the Silymarin group, there was presence of sludge in $16.66 \%$ on the $7^{\text {th }}$ day and in $66.66 \%$ on the $21^{\text {st }}$ day. 
Amaral et al.

Table 1. Mean values of ALT, AST, ALP and GGT of dogs ( $n=6)$ treated for 21 days with starch (400mg), dry extract of Bidens pilosa L. (Asteraceae) (EEB) (100 and 400mg/Kg) and Silymarin $(30 \mathrm{mg} / \mathrm{Kg})$ after oral administration of $\mathrm{CCL}_{4}(2.5 \mathrm{~mL} / \mathrm{Kg})$, Goiânia, Goiás, Brazil

\begin{tabular}{|c|c|c|c|c|c|c|c|c|c|c|c|}
\hline & ALT & BL* & $24 \mathrm{~h}$ & $48 \mathrm{~h}$ & $72 \mathrm{~h}$ & $5 d$ & $8 d$ & $11 d$ & $14 d$ & $17 d$ & $21 d$ \\
\hline \multirow{3}{*}{ Control } & Mean & $38.33^{\mathrm{a}}$ & $266.83^{b}$ & $419.50^{\mathrm{a}}$ & $334.67^{\mathrm{a}}$ & $236.67^{a}$ & $161.40^{\mathrm{a}}$ & $130.10^{\mathrm{a}}$ & $64.86^{\mathrm{a}}$ & $64.20^{\mathrm{a}}$ & $61.60^{a}$ \\
\hline & Std. Dev. & 6.77 & 75.68 & 133.05 & 83.11 & 109.73 & 53.05 & 102.50 & 27.70 & 18.71 & 12.24 \\
\hline & $\mathrm{CV}$ & 17.67 & 28.36 & 31.72 & 24.83 & 46.36 & 32.87 & 78.78 & 42.70 & 29.15 & 19.87 \\
\hline \multirow{3}{*}{$\begin{array}{l}\text { EEB } \\
400 \mathrm{mg} / \mathrm{Kg}\end{array}$} & Mean & $44.08^{\mathrm{a}}$ & $267.33^{b}$ & $253.67^{\mathrm{a}}$ & $138.33^{b}$ & $150.83^{\mathrm{a}}$ & $118.33^{\mathrm{a}}$ & $74.17^{\mathrm{a}}$ & $65.28^{\mathrm{a}}$ & $60.50^{\mathrm{a}}$ & $58.33^{\mathrm{a}}$ \\
\hline & Std. Dev. & 16.79 & 96.64 & 119.46 & 32.98 & 51.09 & 30.49 & 12.86 & 27.80 & 13.34 & 25.86 \\
\hline & $\mathrm{CV}$ & 38.08 & 36.15 & 47.09 & 23.84 & 33.87 & 25.76 & 17.33 & 42.58 & 22.05 & 44.33 \\
\hline \multirow{3}{*}{$\begin{array}{l}\text { EEB } \\
100 \mathrm{mg} / \mathrm{Kg}\end{array}$} & Mean & $42.92^{\mathrm{a}}$ & $276.58^{b}$ & $306.83^{\mathrm{a}}$ & $167.33^{b}$ & $142.33^{\mathrm{a}}$ & $144.67^{\mathrm{a}}$ & $144.92^{\mathrm{a}}$ & $99.33^{\mathrm{a}}$ & $86.33^{\mathrm{a}}$ & $79.67^{\mathrm{a}}$ \\
\hline & Std. Dev. & 10.95 & 83.32 & 75.34 & 79.38 & 49.01 & 48.23 & 68.42 & 61.58 & 36.14 & 40.06 \\
\hline & $\mathrm{CV}$ & 25.52 & 30.13 & 24.55 & 47.44 & 34.44 & 33.34 & 47.21 & 61.99 & 41.86 & 50.28 \\
\hline \multirow{3}{*}{ Silymarin } & Mean & $39.00^{\mathrm{a}}$ & $434.17^{b}$ & $422.33^{b}$ & $224.33^{\mathrm{a}}$ & $182.83^{\mathrm{a}}$ & $159.17^{\mathrm{a}}$ & $98.67^{\mathrm{a}}$ & $71.50^{\mathrm{a}}$ & $67.50^{\mathrm{a}}$ & $61.50^{\mathrm{a}}$ \\
\hline & Std. Dev. & 10.88 & 142.67 & 70.66 & 89.76 & 41.83 & 52.93 & 40.44 & 34.41 & 20.95 & 18.60 \\
\hline & $\mathrm{CV}$ & 27.89 & 32.86 & 16.73 & 40.01 & 22.88 & 33.25 & 40.98 & 48.13 & 31.04 & 30.24 \\
\hline \multirow{4}{*}{ Control } & AST & BL & $24 \mathrm{~h}$ & $48 \mathrm{~h}$ & $72 \mathrm{~h}$ & $5 d$ & $8 d$ & $11 d$ & $14 d$ & $17 d$ & $21 d$ \\
\hline & Mean & $34.17^{\mathrm{a}}$ & $253.50^{\mathrm{a}}$ & $327.00^{\mathrm{a}}$ & $207.92^{\mathrm{a}}$ & $196.67^{\mathrm{a}}$ & $132.00^{\mathrm{a}}$ & $107.40^{\mathrm{a}}$ & $53.00^{\mathrm{a}}$ & $65.20^{\mathrm{a}}$ & $47.60^{\mathrm{a}}$ \\
\hline & Std. Dev. & 13.35 & 106.53 & 89.92 & 65.03 & 122.53 & 53.70 & 26.93 & 13.75 & 35.80 & 7.09 \\
\hline & $\mathrm{CV}$ & 39.08 & 42.02 & 27.50 & 31.28 & 62.30 & 40.68 & 25.08 & 25.94 & 54.91 & 14.90 \\
\hline \multirow{3}{*}{$\begin{array}{l}\text { EEB } \\
400 \mathrm{mg} / \mathrm{Kg}\end{array}$} & Mean & $36.75^{\mathrm{a}}$ & $145.50^{\mathrm{a}}$ & $202.17^{\mathrm{a}}$ & $163.33^{\mathrm{a}}$ & $111.07^{\mathrm{a}}$ & $83.72^{\mathrm{a}}$ & $72.00^{\mathrm{a}}$ & $59.00^{\mathrm{a}}$ & $53.58^{\mathrm{a}}$ & $53.83^{\mathrm{a}}$ \\
\hline & Std. Dev. & 12.11 & 55.50 & 59.52 & 48.36 & 22.86 & 29.78 & 20.78 & 22.10 & 12.94 & 14.69 \\
\hline & $\mathrm{CV}$ & 32.94 & 38.14 & 29.44 & 29.61 & 20.58 & 35.57 & 28.87 & 37.46 & 24.15 & 27.29 \\
\hline \multirow{3}{*}{$\begin{array}{l}\text { EEB } \\
100 \mathrm{mg} / \mathrm{Kg}\end{array}$} & Mean & $35.00^{\mathrm{a}}$ & $148.67^{\mathrm{a}}$ & $252.67^{\mathrm{a}}$ & $155.00^{\mathrm{a}}$ & $114.17^{\mathrm{a}}$ & $77.83^{\mathrm{a}}$ & $91.17^{\mathrm{a}}$ & $78.00^{\mathrm{a}}$ & $74.05^{\mathrm{a}}$ & $49.00^{\mathrm{a}}$ \\
\hline & Std. Dev. & 15.95 & 66.43 & 77.15 & 47.08 & 32.52 & 27.68 & 37.71 & 24.86 & 25.79 & 18.09 \\
\hline & $\mathrm{CV}$ & 45.57 & 44.68 & 30.54 & 30.38 & 28.49 & 35.56 & 41.37 & 31.87 & 34.83 & 36.92 \\
\hline \multirow{4}{*}{ Silymarin } & Mean & $31.92^{\mathrm{a}}$ & $224.17^{\mathrm{a}}$ & $254.50^{\mathrm{a}}$ & $181.37^{\mathrm{a}}$ & $114.67^{\mathrm{a}}$ & $94.33^{\mathrm{a}}$ & $83.92^{\mathrm{a}}$ & $75.83^{\mathrm{a}}$ & $58.25^{\mathrm{a}}$ & $57.67^{\mathrm{a}}$ \\
\hline & Std. Dev. & 7.41 & 63.81 & 32.20 & 27.21 & 43.48 & 36.27 & 28.40 & 19.65 & 37.41 & 31.36 \\
\hline & $\mathrm{CV}$ & 23.20 & 28.47 & 12.65 & 15.00 & 37.92 & 38.45 & 33.84 & 25.91 & 64.22 & 54.38 \\
\hline & ALP & BL & $24 \mathrm{~h}$ & $48 \mathrm{~h}$ & $72 \mathrm{~h}$ & $5 d$ & $8 \mathrm{~d}$ & $11 d$ & $14 d$ & $17 d$ & 21d \\
\hline \multirow{3}{*}{ Control } & Mean & $24.67^{\mathrm{a}}$ & $216.17^{\mathrm{a}}$ & $246.17^{\mathrm{a}}$ & $172.00^{\mathrm{a}}$ & $167.00^{\mathrm{a}}$ & $99.00^{\mathrm{a}}$ & $79.60^{\mathrm{a}}$ & $66.80^{\mathrm{a}}$ & $41.80^{\mathrm{a}}$ & $37.80^{\mathrm{a}}$ \\
\hline & Std. Dev. & 6.98 & 118.67 & 151.16 & 123.91 & 115.31 & 56.86 & 64.46 & 44.21 & 25.16 & 30.58 \\
\hline & $\mathrm{CV}$ & 28.28 & 54.90 & 61.41 & 72.04 & 69.05 & 57.43 & 80.98 & 66.19 & 60.20 & 80.90 \\
\hline \multirow{3}{*}{$\begin{array}{l}\text { EEB } \\
400 \mathrm{mg} / \mathrm{Kg}\end{array}$} & Mean & $27.17^{\mathrm{a}}$ & $185.00^{\mathrm{a}}$ & $174.83^{\mathrm{a}}$ & $125.00^{\mathrm{a}}$ & $99.00^{\mathrm{a}}$ & $60.83^{\mathrm{a}}$ & $60.17^{\mathrm{a}}$ & $47.83^{\mathrm{a}}$ & $36.33^{\mathrm{a}}$ & $32.00^{\mathrm{a}}$ \\
\hline & Std. Dev. & 8.13 & 79.80 & 76.94 & 93.23 & 93.29 & 44.67 & 43.74 & 28.73 & 13.75 & 13.19 \\
\hline & $\mathrm{CV}$ & 29.92 & 43.14 & 44.01 & 74.58 & 94.23 & 73.44 & 72.70 & 60.06 & 37.84 & 41.22 \\
\hline \multirow{3}{*}{$\begin{array}{l}\text { EEB } \\
100 \mathrm{mg} / \mathrm{Kg}\end{array}$} & Mean & $21.33^{\mathrm{a}}$ & $174.17^{\mathrm{a}}$ & $235.67^{\mathrm{a}}$ & $134.00^{\mathrm{a}}$ & $114.50^{\mathrm{a}}$ & $67.17^{\mathrm{a}}$ & $52.50^{\mathrm{a}}$ & $36.67^{\mathrm{a}}$ & $41.17^{\mathrm{a}}$ & $28.83^{\mathrm{a}}$ \\
\hline & Std. Dev. & 4.14 & 46.47 & 102.21 & 39.92 & 49.07 & 38.50 & 17.95 & 7.99 & 16.93 & 8.54 \\
\hline & $\mathrm{CV}$ & 19.42 & 26.68 & 43.37 & 29.79 & 42.85 & 57.32 & 34.20 & 21.80 & 41.12 & 29.63 \\
\hline \multirow{4}{*}{ Silymarin } & Mean & $28.92^{\mathrm{a}}$ & $237.00^{\mathrm{a}}$ & $176.17^{\mathrm{a}}$ & $143.33^{\mathrm{a}}$ & $95.50^{\mathrm{a}}$ & $92.17^{\mathrm{a}}$ & $62.67^{\mathrm{a}}$ & $49.33^{\mathrm{a}}$ & $42.00^{\mathrm{a}}$ & $30.00^{\mathrm{a}}$ \\
\hline & Std. Dev. & 8.05 & 86.21 & 74.04 & 74.93 & 56.79 & 71.45 & 19.22 & 16.08 & 14.32 & 9.59 \\
\hline & $\mathrm{CV}$ & 27.85 & 36.38 & 42.03 & 52.28 & 59.47 & 77.52 & 30.67 & 32.60 & 34.11 & 31.97 \\
\hline & GGT & BL & $24 \mathrm{~h}$ & $48 \mathrm{~h}$ & $72 \mathrm{~h}$ & $5 d$ & $8 \mathrm{~d}$ & $11 d$ & $14 d$ & $17 d$ & $21 d$ \\
\hline \multirow{3}{*}{ Control } & Mean & $6.38^{\mathrm{a}}$ & $11.90^{\mathrm{a}}$ & $12.75^{\mathrm{a}}$ & $8.50^{\mathrm{a}}$ & $9.35^{\mathrm{a}}$ & $6.12^{\mathrm{a}}$ & $9.24^{\mathrm{a}}$ & $6.12^{\mathrm{a}}$ & $6.12^{\mathrm{b}}$ & $8.16^{\mathrm{a}}$ \\
\hline & Std. Dev. & 0.00 & 4.16 & 2.79 & 4.16 & 3.84 & 2.28 & 4.29 & 2.28 & 2.28 & 2.79 \\
\hline & $\mathrm{CV}$ & 0.00 & 34.99 & 21.91 & 48.99 & 41.06 & 37.27 & 46.40 & 37.27 & 37.27 & 34.23 \\
\hline \multirow{3}{*}{$\begin{array}{l}\text { EEB } \\
400 \mathrm{mg} / \mathrm{Kg}\end{array}$} & Mean & $5.95^{\mathrm{a}}$ & $11.05^{\mathrm{a}}$ & $11.90^{\mathrm{a}}$ & $8.50^{\mathrm{a}}$ & $7.65^{\mathrm{a}}$ & $9.35^{\mathrm{a}}$ & $6.80^{\mathrm{a}}$ & $6.80^{\mathrm{a}}$ & $6.80^{\mathrm{b}}$ & $6.80^{\mathrm{a}}$ \\
\hline & Std. Dev. & 0.66 & 3.84 & 2.63 & 2.63 & 2.79 & 3.84 & 2.63 & 2.63 & 2.63 & 2.63 \\
\hline & $\mathrm{CV}$ & 11.07 & 34.74 & 22.13 & 30.98 & 36.51 & 41.06 & 38.73 & 38.73 & 38.73 & 38.73 \\
\hline \multirow{3}{*}{$\begin{array}{l}\text { EEB } \\
100 \mathrm{mg} / \mathrm{Kg}\end{array}$} & Mean & $5.95^{\mathrm{a}}$ & $9.35^{\mathrm{a}}$ & $11.05^{\mathrm{a}}$ & $11.05^{\mathrm{a}}$ & $9.35^{\mathrm{a}}$ & $8.50^{\mathrm{a}}$ & $7.65^{\mathrm{a}}$ & $5.95^{\mathrm{a}}$ & $5.95^{\mathrm{b}}$ & $5.95^{\mathrm{a}}$ \\
\hline & Std. Dev. & 0.66 & 2.08 & 3.84 & 3.84 & 2.08 & 4.16 & 4.27 & 2.08 & 2.08 & 2.08 \\
\hline & $\mathrm{CV}$ & 11.07 & 22.27 & 34.74 & 34.74 & 22.27 & 48.99 & 55.78 & 34.99 & 34.99 & 34.99 \\
\hline \multirow{3}{*}{ Silymarin } & Mean & $6.38^{\mathrm{a}}$ & $8.50^{\mathrm{a}}$ & $12.75^{\mathrm{a}}$ & $11.90^{\mathrm{a}}$ & $7.65^{\mathrm{a}}$ & $9.35^{\mathrm{a}}$ & $8.50^{\mathrm{a}}$ & $9.35^{\mathrm{a}}$ & $6.20^{\mathrm{a}}$ & $8.50^{\mathrm{a}}$ \\
\hline & Std. Dev. & 1.40 & 4.16 & 2.79 & 2.63 & 2.79 & 3.84 & 2.63 & 2.08 & 0.00 & 4.16 \\
\hline & $\mathrm{CV}$ & 21.91 & 48.99 & 21.91 & 22.13 & 36.51 & 41.06 & 30.98 & 22.27 & 0.00 & 48.99 \\
\hline
\end{tabular}


Table 2. Mean values of direct bilirubin (directBB), total bilirubin (totalBB), total proteins (TP), globulin albumins and total cholesterol of $\operatorname{dogs}(\mathrm{n}=6)$ treated for 21 days with starch (negative control), dry extract of Bidens pilosa L. (Asteraceae) (EEB) (100 and $400 \mathrm{mg} / \mathrm{Kg})$ and Silymarin $(30 \mathrm{mg} / \mathrm{Kg})$ after oral administration of $\mathrm{CCL}_{4}(2.5 \mathrm{~mL} / \mathrm{Kg})$, Goiânia, Goiás, Brazil

\begin{tabular}{|c|c|c|c|c|c|c|c|c|c|c|c|}
\hline & totalBB & BL* & $24 \mathrm{~h}$ & $48 \mathrm{~h}$ & $72 \mathrm{~h}$ & $5 d$ & $8 d$ & $11 \mathrm{~d}$ & $14 d$ & $17 \mathrm{~d}$ & 21d \\
\hline \multirow{3}{*}{ Control } & Mean & $0.72^{\mathrm{a}}$ & $1.06^{\mathrm{a}}$ & $1.08^{\mathrm{a}}$ & $1.20^{\mathrm{a}}$ & $1.27^{\mathrm{a}}$ & $1.06^{\mathrm{a}}$ & $1.18^{\mathrm{b}}$ & $0.97^{\mathrm{a}}$ & $1.02^{\mathrm{a}}$ & $1.00^{\mathrm{a}}$ \\
\hline & Std. Dev. & 0.15 & 0.17 & 0.12 & 0.42 & 0.46 & 0.17 & 0.15 & 0.24 & 0.19 & 0.20 \\
\hline & $\mathrm{CV}$ & 20.47 & 16.43 & 10.85 & 34.96 & 36.58 & 15.79 & 12.57 & 24.76 & 18.22 & 20.29 \\
\hline \multirow{3}{*}{$\begin{array}{l}\text { EEB } \\
400 \mathrm{mg} / \mathrm{Kg}\end{array}$} & Mean & $0.62^{\mathrm{a}}$ & $1.04^{\mathrm{a}}$ & $0.97^{\mathrm{a}}$ & $0.94^{\mathrm{a}}$ & $0.94^{\mathrm{a}}$ & $0.86^{\mathrm{a}}$ & $0.88^{\mathrm{a}}$ & $0.83^{\mathrm{a}}$ & $0.82^{\mathrm{a}}$ & $0.87^{\mathrm{a}}$ \\
\hline & Std. Dev. & 0.04 & 0.10 & 0.16 & 0.20 & 0.12 & 0.12 & 0.23 & 0.20 & 0.21 & 0.24 \\
\hline & $\mathrm{CV}$ & 6.58 & 9.48 & 16.91 & 21.53 & 12.84 & 14.31 & 26.23 & 24.17 & 25.44 & 28.26 \\
\hline \multirow{3}{*}{$\begin{array}{l}\text { EEB } \\
100 \mathrm{mg} / \mathrm{Kg}\end{array}$} & Mean & $0.73^{\mathrm{a}}$ & $0.88^{\mathrm{a}}$ & $0.89^{\mathrm{a}}$ & $0.98^{\mathrm{a}}$ & $0.80^{\mathrm{a}}$ & $0.91^{\mathrm{a}}$ & $0.90^{\mathrm{b}}$ & $0.89^{\mathrm{a}}$ & $0.87^{\mathrm{a}}$ & $0.79^{\mathrm{a}}$ \\
\hline & Std. Dev. & 0.15 & 0.16 & 0.19 & 0.12 & 0.14 & 0.10 & 0.10 & 0.09 & 0.12 & 0.24 \\
\hline & $\mathrm{CV}$ & 20.99 & 17.90 & 20.87 & 11.89 & 17.68 & 11.10 & 11.58 & 10.29 & 13.58 & 29.97 \\
\hline \multirow{4}{*}{ Silymarin } & Mean & $0.68^{\mathrm{a}}$ & $1.07^{\mathrm{a}}$ & $1.00^{\mathrm{a}}$ & $0.99^{\mathrm{a}}$ & $0.82^{\mathrm{a}}$ & $0.94^{\mathrm{a}}$ & $0.90^{\mathrm{b}}$ & $0.82^{\mathrm{a}}$ & $0.82^{\mathrm{a}}$ & $0.80^{\mathrm{a}}$ \\
\hline & Std. Dev. & 0.17 & 0.18 & 0.15 & 0.08 & 0.28 & 0.18 & 0.17 & 0.18 & 0.16 & 0.14 \\
\hline & $\mathrm{CV}$ & 25.44 & 16.53 & 14.52 & 8.28 & 34.12 & 18.81 & 18.59 & 22.54 & 19.62 & 16.93 \\
\hline & directBB & $\mathrm{BL}$ & $24 \mathrm{~h}$ & $48 \mathrm{~h}$ & $72 \mathrm{~h}$ & $5 d$ & $8 d$ & $11 \mathrm{~d}$ & $14 d$ & $17 d$ & $21 d$ \\
\hline \multirow{3}{*}{ Control } & Mean & $0.19^{a}$ & $0.60^{\mathrm{a}}$ & $0.36^{\mathrm{a}}$ & $0.40^{\mathrm{a}}$ & $0.30^{\mathrm{a}}$ & $0.32^{\mathrm{a}}$ & $0.38^{\mathrm{a}}$ & $0.35^{\mathrm{a}}$ & $0.39^{\mathrm{a}}$ & $0.36^{\mathrm{a}}$ \\
\hline & Std. Dev. & 0.07 & 0.25 & 0.08 & 0.12 & 0.31 & 0.10 & 0.05 & 0.12 & 0.11 & 0.03 \\
\hline & $\mathrm{CV}$ & 35.92 & 41.49 & 23.40 & 30.89 & 0.30 & 30.91 & 12.46 & 34.39 & 27.96 & 9.02 \\
\hline \multirow{3}{*}{$\begin{array}{l}\text { EEB } \\
400 \mathrm{mg} / \mathrm{Kg}\end{array}$} & Mean & $0.20^{\mathrm{a}}$ & $0.37^{\mathrm{a}}$ & $0.31^{\mathrm{a}}$ & $0.35^{\mathrm{a}}$ & $0.30^{\mathrm{a}}$ & $0.27^{\mathrm{a}}$ & $0.25^{\mathrm{b}}$ & $0.32^{\mathrm{a}}$ & $0.29^{\mathrm{a}}$ & $0.31^{\mathrm{a}}$ \\
\hline & Std. Dev. & 0.07 & 0.09 & 0.03 & 0.07 & 0.05 & 0.06 & 0.07 & 0.10 & 0.11 & 0.08 \\
\hline & $\mathrm{CV}$ & 37.52 & 24.33 & 8.71 & 21.25 & 17.79 & 23.66 & 29.75 & 32.27 & 36.69 & 25.88 \\
\hline \multirow{3}{*}{$\begin{array}{l}\text { EEB } \\
100 \mathrm{mg} / \mathrm{Kg}\end{array}$} & Mean & $0.17^{\mathrm{a}}$ & $0.47^{\mathrm{a}}$ & $0.33^{\mathrm{a}}$ & $0.33^{\mathrm{a}}$ & $0.32^{\mathrm{a}}$ & $0.31^{\mathrm{a}}$ & $0.32^{\mathrm{a}}$ & $0.38^{\mathrm{a}}$ & $0.39^{\mathrm{a}}$ & $0.36^{\mathrm{a}}$ \\
\hline & Std. Dev. & 0.08 & 0.08 & 0.06 & 0.07 & 0.05 & 0.05 & 0.04 & 0.08 & 0.10 & 0.07 \\
\hline & $\mathrm{CV}$ & 45.52 & 16.55 & 18.30 & 21.83 & 16.55 & 17.81 & 12.81 & 21.96 & 25.07 & 18.79 \\
\hline \multirow{4}{*}{ Silymarin } & Mean & $0.21^{\mathrm{a}}$ & $0.43^{\mathrm{a}}$ & $0.35^{\mathrm{a}}$ & $0.34^{\mathrm{a}}$ & $0.48^{\mathrm{a}}$ & $0.33^{\mathrm{a}}$ & $0.33^{\mathrm{a}}$ & $0.33^{\mathrm{a}}$ & $0.28^{\mathrm{a}}$ & $0.28^{\mathrm{a}}$ \\
\hline & Std. Dev. & 0.07 & 0.19 & 0.05 & 0.09 & 0.24 & 0.29 & 0.08 & 0.06 & 0.05 & 0.05 \\
\hline & $\mathrm{CV}$ & 35.21 & 44.59 & 15.55 & 27.02 & 50.74 & 88.63 & 23.39 & 17.60 & 19.16 & 16.75 \\
\hline & $\mathrm{TP}$ & $\mathrm{BL}$ & $24 \mathrm{~h}$ & $48 \mathrm{~h}$ & $72 \mathrm{~h}$ & $5 d$ & $8 \mathrm{~d}$ & $11 d$ & $14 d$ & $17 d$ & $21 d$ \\
\hline \multirow{3}{*}{ Control } & Mean & $6.48^{\mathrm{a}}$ & $7.22^{\mathrm{a}}$ & $7.03^{\mathrm{a}}$ & $7.43^{\mathrm{a}}$ & $7.53^{\mathrm{a}}$ & $7.74^{\mathrm{a}}$ & $7.44^{\mathrm{a}}$ & $7.38^{\mathrm{a}}$ & $7.90^{\mathrm{a}}$ & $7.50^{\mathrm{a}}$ \\
\hline & Std. Dev. & 0.48 & 0.92 & 0.68 & 0.85 & 0.86 & 0.53 & 0.44 & 1.00 & 0.62 & 0.72 \\
\hline & $\mathrm{CV}$ & 7.46 & 12.71 & 9.63 & 11.47 & 11.47 & 6.87 & 5.90 & 13.50 & 7.85 & 9.66 \\
\hline \multirow{3}{*}{$\begin{array}{l}\text { EEB } \\
400 \mathrm{mg} / \mathrm{Kg}\end{array}$} & Mean & $6.75^{\mathrm{a}}$ & $6.72^{\mathrm{a}}$ & $6.42^{\mathrm{a}}$ & $6.85^{\mathrm{a}}$ & $7.22^{\mathrm{a}}$ & $7.00^{\mathrm{a}}$ & $7.08^{\mathrm{a}}$ & $7.07^{\mathrm{a}}$ & $7.23^{\mathrm{a}}$ & $7.30^{\mathrm{a}}$ \\
\hline & Std. Dev. & 0.38 & 0.81 & 0.50 & 0.69 & 0.97 & 0.50 & 0.60 & 0.73 & 0.48 & 0.51 \\
\hline & $\mathrm{CV}$ & 5.68 & 12.00 & 7.85 & 10.15 & 13.39 & 7.11 & 8.54 & 10.31 & 6.58 & 6.93 \\
\hline \multirow{3}{*}{$\begin{array}{l}\text { EEB } \\
100 \mathrm{mg} / \mathrm{Kg}\end{array}$} & Mean & $6.77^{\mathrm{a}}$ & $7.03^{\mathrm{a}}$ & $7.02^{\mathrm{a}}$ & $7.18^{\mathrm{a}}$ & $7.32^{\mathrm{a}}$ & $7.38^{\mathrm{a}}$ & $7.55^{\mathrm{a}}$ & $7.52^{\mathrm{a}}$ & $7.63^{\mathrm{a}}$ & $8.03^{\mathrm{a}}$ \\
\hline & Std. Dev. & 0.49 & 0.94 & 1.02 & 0.66 & 0.81 & 1.02 & 0.55 & 0.89 & 1.06 & 0.46 \\
\hline & $\mathrm{CV}$ & 7.28 & 13.33 & 14.55 & 9.13 & 11.08 & 13.80 & 7.24 & 11.82 & 13.93 & 5.71 \\
\hline \multirow{4}{*}{ Silymarin } & Mean & $6.15^{\mathrm{a}}$ & $6.35^{\mathrm{a}}$ & $6.37^{\mathrm{a}}$ & $6.95^{\mathrm{a}}$ & $7.37^{\mathrm{a}}$ & $6.88^{\mathrm{a}}$ & $6.90^{\mathrm{a}}$ & $6.82^{\mathrm{a}}$ & $6.97^{\mathrm{a}}$ & $6.83^{\mathrm{a}}$ \\
\hline & Std. Dev. & 0.53 & 0.48 & 0.94 & 0.46 & 0.94 & 0.60 & 0.27 & 0.44 & 0.84 & 0.75 \\
\hline & $\mathrm{CV}$ & 8.65 & 7.50 & 14.82 & 6.61 & 12.78 & 8.74 & 3.89 & 6.39 & 12.07 & 10.98 \\
\hline & Albumin & $\mathrm{BL}$ & $24 \mathrm{~h}$ & $48 \mathrm{~h}$ & $72 \mathrm{~h}$ & $5 d$ & $8 \mathrm{~d}$ & $11 \mathrm{~d}$ & $14 d$ & $17 d$ & $21 d$ \\
\hline & Mean & $2.88^{\mathrm{a}}$ & $2.75^{\mathrm{a}}$ & $3.23^{\mathrm{a}}$ & $3.05^{\mathrm{a}}$ & $2.68^{\mathrm{a}}$ & $2.81^{\mathrm{a}}$ & $2.99^{\mathrm{a}}$ & $3.04^{\mathrm{a}}$ & $2.98^{\mathrm{a}}$ & $2.51^{\mathrm{a}}$ \\
\hline Control & Std. Dev. & 0.26 & 0.54 & 0.76 & 0.27 & 0.34 & 0.47 & 0.58 & 0.38 & 0.37 & 0.31 \\
\hline & $\mathrm{CV}$ & 9.05 & 19.58 & 23.51 & 8.94 & 12.77 & 16.60 & 19.25 & 12.41 & 12.52 & 12.37 \\
\hline & Mean & $3.09^{\mathrm{a}}$ & $2.82^{\mathrm{a}}$ & $2.92^{\mathrm{a}}$ & $3.36^{\mathrm{a}}$ & $2.79^{\mathrm{a}}$ & $3.05^{\mathrm{a}}$ & $2.69^{\mathrm{a}}$ & $2.77^{\mathrm{a}}$ & $2.99^{\mathrm{a}}$ & $2.98^{\mathrm{a}}$ \\
\hline EEB & Std. Dev. & 0.30 & 0.46 & 0.71 & 0.56 & 0.18 & 0.32 & 0.39 & 0.51 & 0.36 & 0.25 \\
\hline $400 \mathrm{mg} / \mathrm{Kg}$ & $\mathrm{CV}$ & 9.73 & 16.26 & 24.44 & 16.55 & 6.45 & 10.66 & 14.52 & 18.41 & 12.12 & 8.56 \\
\hline & Mean & $3.00^{\mathrm{a}}$ & $3.18^{\mathrm{a}}$ & $3.46^{\mathrm{a}}$ & $3.04^{\mathrm{a}}$ & $3.16^{\mathrm{a}}$ & $3.07^{\mathrm{a}}$ & $2.68^{\mathrm{a}}$ & $2.99^{\mathrm{a}}$ & $2.97^{\mathrm{a}}$ & $3.05^{\mathrm{a}}$ \\
\hline $\begin{array}{l}\text { EEB } \\
100 \mathrm{mg} / \mathrm{Kg}\end{array}$ & Std. Dev. & 0.23 & 0.31 & 1.10 & 0.33 & 0.50 & 0.51 & 0.26 & 0.32 & 0.37 & 0.33 \\
\hline $100 \mathrm{mg} / \mathrm{Kg}$ & $\mathrm{CV}$ & 7.52 & 9.72 & 31.84 & 10.69 & 15.77 & 16.70 & 9.87 & 10.59 & 12.61 & 10.84 \\
\hline & Mean & $2.77^{\mathrm{a}}$ & $2.65^{\mathrm{a}}$ & $3.16^{\mathrm{a}}$ & $2.90^{\mathrm{a}}$ & $2.97^{\mathrm{a}}$ & $2.94^{\mathrm{a}}$ & $3.05^{\mathrm{a}}$ & $3.22^{\mathrm{a}}$ & $3.02^{\mathrm{a}}$ & $2.86^{\mathrm{a}}$ \\
\hline Silymarin & Std. Dev. & 0.25 & 0.40 & 1.44 & 0.34 & 0.38 & 0.23 & 0.49 & 0.35 & 0.48 & 0.23 \\
\hline & $\mathrm{CV}$ & 8.88 & 15.28 & 45.64 & 11.60 & 12.76 & 7.66 & 16.09 & 10.84 & 16.04 & 8.20 \\
\hline & Globulins & $\mathrm{BL}$ & $24 \mathrm{~h}$ & $48 \mathrm{~h}$ & $72 \mathrm{~h}$ & $5 d$ & $8 d$ & $11 d$ & $14 d$ & $17 d$ & $21 d$ \\
\hline & Mean & $3.60^{\mathrm{a}}$ & $4.46^{\mathrm{a}}$ & $3.81^{\mathrm{a}}$ & $4.39^{\mathrm{a}}$ & $4.85^{\mathrm{a}}$ & $4.11^{\mathrm{a}}$ & $3.71^{\mathrm{a}}$ & $3.62^{\mathrm{a}}$ & $4.10^{\mathrm{a}}$ & $4.16^{\mathrm{a}}$ \\
\hline Control & Std. Dev. & 0.52 & 1.41 & 0.90 & 0.86 & 1.08 & 2.05 & 1.94 & 1.87 & 2.18 & 2.22 \\
\hline Control & $\mathrm{CV}$ & 14.39 & 31.56 & 23.61 & 19.62 & 22.27 & 49.94 & 52.30 & 51.82 & 53.19 & 53.39 \\
\hline & $\mathrm{CV}$ & 5.95 & 25.31 & 23.76 & 29.68 & 19.12 & 17.21 & 22.03 & 15.22 & 11.38 & 10.06 \\
\hline & Mean & $3.77^{\mathrm{a}}$ & $3.86^{\mathrm{a}}$ & $3.56^{\mathrm{a}}$ & $4.14^{\mathrm{a}}$ & $4.16^{\mathrm{a}}$ & $4.31^{\mathrm{a}}$ & $4.88^{\mathrm{a}}$ & $4.53^{\mathrm{a}}$ & $4.67^{\mathrm{a}}$ & $4.98^{\mathrm{a}}$ \\
\hline EEB & Std. Dev. & 0.39 & 0.71 & 1.72 & 0.71 & 1.09 & 0.72 & 0.57 & 1.06 & 1.23 & 0.66 \\
\hline $100 \mathrm{mg} / \mathrm{Kg}$ & $\mathrm{CV}$ & 10.39 & 18.47 & 48.35 & 17.15 & 26.28 & 16.65 & 11.63 & 23.33 & 26.38 & 13.18 \\
\hline & Mean & $3.38^{\mathrm{a}}$ & $3.71^{\mathrm{a}}$ & $3.21^{\mathrm{a}}$ & $4.05^{\mathrm{a}}$ & $4.40^{\mathrm{a}}$ & $3.94^{\mathrm{a}}$ & $3.85^{\mathrm{a}}$ & $3.60^{\mathrm{a}}$ & $3.95^{\mathrm{a}}$ & $3.97^{\mathrm{a}}$ \\
\hline Silymarın & Std. Dev. & 0.65 & 0.80 & 2.00 & 0.65 & 0.79 & 0.52 & 0.52 & 0.75 & 0.93 & 0.81 \\
\hline
\end{tabular}


Amaral et al.

\begin{tabular}{llllllllllll} 
& CV & 19.26 & 21.72 & 62.21 & 15.99 & 17.87 & 13.12 & 13.58 & 20.74 & 23.56 & 20.46 \\
& Cholesterol & BL & $24 \mathrm{~h}$ & $48 \mathrm{~h}$ & $72 \mathrm{~h}$ & $5 \mathrm{~d}$ & $8 \mathrm{~d}$ & $11 \mathrm{~d}$ & $14 \mathrm{~d}$ & $17 \mathrm{~d}$ & $21 \mathrm{~d}$ \\
& Mean & $170.25^{\mathrm{a}}$ & $200.17^{\mathrm{a}}$ & $147.17^{\mathrm{a}}$ & $183.50^{\mathrm{a}}$ & $208.67^{\mathrm{a}}$ & $185.20^{\mathrm{a}}$ & $223.80^{\mathrm{a}}$ & $244.00^{\mathrm{a}}$ & $248.00^{\mathrm{a}}$ & $211.20^{\mathrm{a}}$ \\
Control & Std. Dev. & 25.03 & 52.83 & 27.92 & 49.71 & 66.96 & 24.17 & 65.49 & 32.76 & 53.61 & 32.81 \\
& CV & 14.70 & 26.39 & 18.97 & 27.09 & 32.09 & 13.05 & 29.26 & 13.42 & 21.62 & 15.54 \\
& Mean & $175.00^{\mathrm{a}}$ & $176.83^{\mathrm{a}}$ & $161.33^{\mathrm{a}}$ & $195.00^{\mathrm{a}}$ & $210.00^{\mathrm{a}}$ & $206.00^{\mathrm{a}}$ & $233.17^{\mathrm{a}}$ & $217.67^{\mathrm{a}}$ & $220.83^{\mathrm{a}}$ & $196.00^{\mathrm{a}}$ \\
EEB & Std. Dev. & 32.78 & 59.17 & 31.95 & 13.51 & 84.10 & 56.59 & 46.01 & 46.31 & 64.66 & 39.31 \\
$400 \mathrm{mg} / \mathrm{Kg}$ & CV & 18.73 & 33.46 & 19.80 & 6.93 & 40.05 & 27.47 & 19.73 & 21.27 & 29.28 & 20.06 \\
& Mean & $186.67^{\mathrm{a}}$ & $196.17^{\mathrm{a}}$ & $175.67^{\mathrm{a}}$ & $207.17^{\mathrm{a}}$ & $244.17^{\mathrm{a}}$ & $253.67^{\mathrm{a}}$ & $221.33^{\mathrm{a}}$ & $192.83^{\mathrm{a}}$ & $213.00^{\mathrm{a}}$ & $210.33^{\mathrm{a}}$ \\
EEB & Std. Dev. & 24.71 & 71.41 & 37.56 & 59.23 & 88.60 & 142.97 & 36.19 & 46.15 & 74.73 & 53.85 \\
$100 \mathrm{mg} / \mathrm{Kg}$ & CV & 13.24 & 36.40 & 21.38 & 28.59 & 36.29 & 56.36 & 16.35 & 23.93 & 35.09 & 25.60 \\
& Mean & $186.58^{\mathrm{a}}$ & $168.17^{\mathrm{a}}$ & $199.17^{\mathrm{a}}$ & $196.67^{\mathrm{a}}$ & $193.17^{\mathrm{a}}$ & $157.50^{\mathrm{a}}$ & $208.17^{\mathrm{a}}$ & $257.00^{\mathrm{a}}$ & $236.83^{\mathrm{a}}$ & $203.00^{\mathrm{a}}$ \\
\multirow{3}{*}{ Silymarin } & Std. Dev. & 36.15 & 49.29 & 69.68 & 56.82 & 68.10 & 62.45 & 52.88 & 54.71 & 45.76 & 71.62 \\
& CV & 19.37 & 29.31 & 34.99 & 28.89 & 35.26 & 39.65 & 25.40 & 21.29 & 19.32 & 35.28 \\
\hline
\end{tabular}

$\mathrm{BL}-$ Base Line. Equal letters in the same column do not differ between themselves (ANOVA and $t$. Test, $\mathrm{P}<0.05$ ).

The histological analysis of samples collected 48 hours after intoxication revealed the presence of microvacuolar hydropic degeneration of hepatocytes and bile ducts and necrosis (Figure 1). Generally, necrosis was observed in isolated hepatocytes or in small groups, especially in areas close to the central vein. Other changes observed were: presence of lymphoplasmocytic and eosinophilic inflammatory infiltrate, congestion of blood vessels and atherosclerosis (Figure 1). On the $7^{\text {th }}$ day after the intoxication, the persistence of histological changes was observed. However, in the animals treated with EEB at a dose of $400 \mathrm{mg} / \mathrm{Kg}$, lower incidence of changes was observed, when compared to the other groups, however, significant differences $(\mathrm{P}>0.05)$ were not detected. On the $21^{\text {st }}$ day of analysis, the samples collected for histopathological analysis showed no significant changes, with adequate recovery of the liver tissue in all experimental groups.

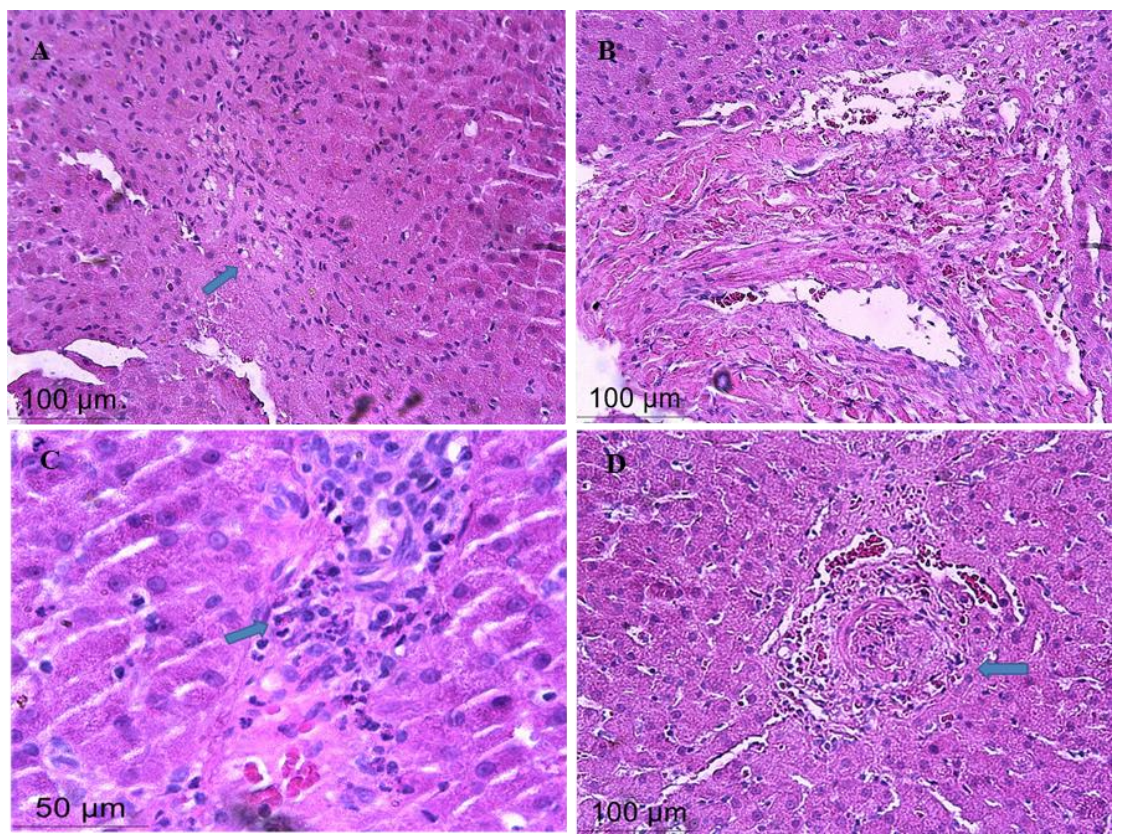

Figure 1. Photomicrograph of a hydropic degeneration area of hepatocytes and necrosis in a liver of control group dog (A); microvacuolar degeneration and hepatocyte and blood vessel necrosis in the liver of a dog of the group treated with ethanolic extract of Bidens pilosa L. (Asteraceae) at a dose of 100 $\mathrm{mg} / \mathrm{kg}$ (B); focal degeneration of the bile duct and inflammatory infiltrate near a bile duct in a dog of the group treated with silymarin (C); cholangitis in a dog treated with ethanolic extract of Bidens pilosa L. (Asteraceae) at a dose of $400 \mathrm{mg} / \mathrm{kg}$ (D), 48 hours after intoxication by $\mathrm{CCl}_{4}$ in a single oral dose of 2.5mL/Kg. HE, 20X. Goiânia, Goiás, Brazil. 


\section{DISCUSSION}

Overall, the clinical, biochemical, ultrasound and histological changes observed after the intoxication with carbon tetrachloride in the different experimental groups were similar and reversible, corroborating the results reported in the literature (Aguilera-Tejelo, 1988; Assy and Minuk, 1997) which used a similar methodology for the induction of toxic liver injuries in dogs. The use of $\mathrm{CCl}_{4}$ as intermediate in chemical reactions was severely restricted due to its high toxicity. However, once it induces hepatotoxicity tissue, enzymatic and clinic changes, the $\mathrm{CCl}_{4}$ has been used in experimental protocols to elucidate the mechanisms of acute and chronic hepatitis and their treatments (Weber et al., 2003).

Clinical signs observed in animals after the oral administration of $\mathrm{CCL}_{4}$, although nonspecific, are consistent with the clinical condition of acute hepatotoxicity. According to Rogers (1986), anorexia, depression, vomiting and diarrhea, may be observed in dogs with hepatotoxicity by $\mathrm{CCl}_{4}$. According to Center (1999), in acute liver failure, especially in those caused by xenobiotics, diarrhea could be observed, as noticed in some $\operatorname{dogs}$ of this study. Despite no significant difference was generally observed among the groups treated, a lower incidence of clinical signs was noticed in the groups treated with EEB at a dose of $100 \mathrm{mg} / \mathrm{Kg}$, followed by the animals that received EEB at a dose of $400 \mathrm{mg} / \mathrm{Kg}$ and those treated with Silymarin. The animals of the control group showed a higher number of episodes of vomiting and diarrhea.

Specifically, the increased plasma concentration of ALT, an enzyme present in the cytosol, results from the membrane permeability, and consequently, indicates cell death (Center, 1999; Giannini et al., 2005; Grigorescu, 2006). The hepatotoxic effect of $\mathrm{CCL}_{4}$ is related to cell death by necrosis and/or induction of apoptosis, which could justify the increased serum activity of ALT (Golstein and Kroemer, 2012). In this study, the lowest serum activities of ALT after intoxication were observed in the groups treated with Bidens pilosa extract, indicating that the extract showed hepatoprotective effect against lipid peroxidation, an important mechanism of protection against acute liver injury. This finding corroborates the study by Chin et al. (1996) conducted in rats, where the ethanolic extract of this plant species showed hepatoprotective effect in mice intoxicated acutely by $\mathrm{CCl}_{4}$.

Silymarin acts in the prevention of liver injuries induced by different chemicals and toxins (Lieber et al., 2003; Shaker et al., 2011; Li et al., 2012). In this study, the Silymarin showed significant increased serum activity of ALT after acute intoxication in the group treated, indicating that this compound did not show hepatoprotective action in the first 24 hours after liver injury. On the other hand, a hepatoprotective effect of the Silymarin administered for six days after the hepatic injury was observed in other studies (Lieber et al., 2003; Shaker et al., 2011).

The dogs treated with Bidens pilosa extract and Silymarin showed the lowest serum concentrations of total bilirubin and, therefore, acted positively on the hepatocytes. A similar result for Silymarin was reported by Yadav et al. (2008) in a study developed in rats. Whereas direct bilirubin reflects the conjugation ability of the hepatocytes (Giannini et al., 2005), it could be observed that the Bidens pilosa extract reestablished the liver conjugation process more quickly, when compared to the Silymarin and control groups, at all times evaluated, after injury with carbon tetrachloride. No other studies were found on the effect of Bidens pilosa on serum concentration of bilirubin in dogs.

The ultrasound aspect of the liver of dogs before the intoxication was consistent with that of a healthy organ, whereas the description by Nyland et al. (2005), because it showed regular contour and size, hyperechoic or isoecogenic parenchyma in relation to the renal cortex, homogeneous echotexture and no signs of biliary sludge. Liver ultrasound changes were observed with higher frequency on the $7^{\text {th }}$ day after the intoxication, as well as histological changes, in all experimental groups. Sen et al. (2005) obtained similar results and reported that the dogs experimentally intoxicated by $\mathrm{CCL}_{4}$ showed significant ultrasound changes on the $5^{\text {th }}$ day. At the end of the evaluation period, the liver size of most $(70.83 \%)$ dogs returned to normal in all experimental groups, as described by other authors who induced the acute hepatotoxicity in dogs experimentally after a single dose of carbon tetrachloride (Sen et al., 2005). 
On histological evaluation, necrosis in isolated hepatocytes or in small groups was observed, especially in areas close to the central vein. Other changes observed were: presence of lymphoplasmocytic and eosinophilic inflammatory infiltrate, congestion of blood vessels and atherosclerosis. These changes were observed uniformly when among the groups and are in accordance with those reported by Sen et al. (2005) in dogs intoxicated with $\mathrm{CCL}_{4}$. On the $7^{\text {th }}$ day after the intoxication, the persistence of the histological changes described could be observed, with no significant statistical difference among the groups. Already on the $21^{\text {st }}$ day of analysis, the samples collected for the histopathological analysis showed no significant changes, with adequate recovery of liver tissue in all experimental groups. The result is also in accordance with those found by Sen et al. (2005).

\section{CONCLUSION}

The dry extract of Bidens pilosa proved to be more efficient than Silymarin in the treatment of acute toxic hepatitis induced in dogs.

\section{REFERENCES}

AGUILERA-TEJERO, E. Plasma bile acids, lactate dehydrogenase and sulfobrophthalein retention test in canine carbon tetrachloride intoxication. J. Small Anim. Pract., v.29, p.711717, 1988.

ALONSO, J.R. Tratado de fitofármacos $e$ nutracêuticos. São Paulo: AC Farmacêutica, 2016. 1124p.

ASGHAR, Z.; MASOOD, Z. Evaluation of antioxidant properties of silymarin and its potential to inhibit peroxyl radicals in vitro. Pak. J. Pharm. Sci., v.21, p.249-254, 2008.

ASSY, N.; MINUK, G.Y. Liver regeneration: methods for monitoring and their applications. $J$. Hepatol., v.26, p.945-52, 1997.

BARTOLOME, A.P.; VILLASEÑOR, I.M.; YANG, W.C. Bidens pilosa L. (asteraceae): botanical properties, traditional uses, phytochemistry, and pharmacology. Evidence Based Complemen. Altern. Med., v.2013, p.34021, 2013.
BASU, S. Carbon tetrachloride-induced lipid peroxidation: eicosanoid formation and their regulation by antioxidant nutrients. Toxicology, v.189, p.113-127, 2003.

BRASIL. Ministério da Saúde. Monografia da Espécie Bidens pilosa (Picão-preto). 2015. Disponível em: </portalarquivos2.saude.gov.br/images/pdf/2017/ setembro/11/ Monografia-Bidens.pdf. Acessado em: 11 dez. 2019.

CENTER, S.A. Chronic liver disease: current concepts of disease mechanisms. J. Small Anim. Pract., v.40, p.106-114,1999.

CHEN, A.S.; TAGUCHI, T.; SAKA, K. et al. Effect of chitobiose and chitotriose on carbon tetrachloride-induced acute hepatotoxicity in rats. Biol. Pharm. Bull., v.28, p.1971-1973, 2005.

CHIN, H.W.; LIN, C.C.; TANG, K. The hepatoprotective effects of Taiwan folk medicine ham-hong-chcho in rats. Am. J. Med. Chin., v.24, p.231-240, 1996.

EL-KAMARY, S.S.; SHARDELL, M.D.; ABDEL-HAMID, M. et al. A randomized controlled trial to assess the safety and efficacy of silymarin on symptoms, signs and biomarkers of acute hepatitis. Phytomedicine, v.16, p.391400, 2009.

GIANNINI, E.G.; TESTA, R.; SAVARINO, V. Liver enzyme alteration: a guide for clinicians. Can. Med. Assoc. J., v.172, p.367-379. 2005.

GOLSTEIN, P.; KROEMER, G. Cell death by necrosis: towards a molecular definition. Trends Biochem. Sci., v.32, p.37-43, 2007

GRIGORESCU, M. Noninvasive biochemical makers of liver fibrosis. J. Gastrointestin. Liver Dis., v.15, p.149-159, 2006.

KRISHNAIAH, D.; SARBATLY, R.; NITHYANANDAM, R. A review of the antioxidant potential of medicinal plants species. Food Bioprod. Proces., v.89, p.217-233, 2011.

LI, C.C.; HSIANG, C.Y.; WUC, S.L. et al. Identification of novel mechanisms of silymarin on the carbontetrachloride-induced liver fibrosis in mice by nuclear factor-Jb bioluminescent imaging-guided transcriptomic analysis. Food Chem. Toxicol., v.50, p.1568-1575. 2012. 
LIEBER, C.S.; LEO, M.A.; CAO, Q. et al. Silymarin retards the progression of alcoholinduced hepatic fibrosis in baboons. J. Clin. Gastroenterol., v.37, p.336-339, 2003.

LORENZI, H.; MATOS, F.J A. Plantas medicinais do Brasil: nativas e exóticas. 3.ed. Nova Odessa, SP: Instituto Plantarum, 2008. $674 \mathrm{p}$.

NYLAND, T.G.; MATTOON, J.S.; HERRGESELL, E.J. et al. Fígado. Ultrassom diagnóstico em pequenos animais. 2.ed. São Paulo: Roca, 2005. 400p.

ROGERS, W.A. Disease of the liver. In: JONES, B.D.; LISKA, W.D. Canine and feline gastroenterology. Philadelphia: WB Saunders, 1986. 678p.

ROLIM, A.; MACIEL, C.P.M.; KANEKO, T.M. et al. Validation assay for total flavonoids, as rutin equivalents, from Trichilia catigua Adr.Juss (Meliaceae) and Ptychopetalum olacoides Bentham (Olacaceae) commercial extract. $J$. AOAC Int., v.88, p.1015-1019, 2005.

SAMPAIO, I.B.M. Estatística aplicada à experimentação animal. Belo Horizonte: Fundação de Ensino e Pesquisa em Medicina Veterinária e Zootecnia, 2002. 221p.

SANTOS FILHO, E.X.S.; ARANTES, D.A.C.; LEITE, A.F.O et al. Randomized clinical trial of a mucoadhesive formulation containing curcuminoids (Zingiberaceae) and Bidens pilosa Linn (Asteraceae) extract (FITOPROT) for prevention and treatment of oral mucositis phase I study. Chem. Biolog. Int., v.291, p.228236, 2018.

SCHULZE-OSTHOFF, K.; HAUSSINGER, D. Apoptosis in the liver: a matter of ion fluxes and oxidative stress. Liver Int., v.27, p.1039-1044, 2007.
SEN, I.; TURGUT, M.; KIRAN, M.; OK, M.M. et al. Effects of nutritional therapy or n-AcetylCysteine (NAC) treatment on biochemical markers and liver histology in dogs with $\mathrm{CCL}_{4-}$ induced hepatic necrosis. Rev. Méd. Vét., v.156, p.483-490, 2005.

SHAKER, M.E.; SHIHA, G.E.; IBRAHIM, T.M. Comparison of early treatment with low doses of nilotinib, imatinib and a clinically relevant dose of silymarin in thioacetamide-induced liver fibrosis. Eur. J. Pharmacol., v.670, p.593-600, 2011.

SILVA, F.L.; FISCHER, D.C.H.; TAVARES, J.F. et al. Compilation of secondary metabolites from Bidens pilosa L, Molecules, v.16, p.10701102, 2011.

TSAI, M.J.; LIAO, J.F.; LIN, D.Y. et al. Silymarin protects spinal cord and cortical cells against oxidative stress and lipopolysaccharide stimulation. Neurochem. Int., v.57, p.867-875, 2010 .

WEBER, L.W.D.; BOLL, M.; STAMPFL, A. Hepatotoxity and mechanism of action of haloalkanes: carbon tetrachloride as a toxicological model. Crit. Rev. Toxicol., v.33, p.105-136, 2003.

YADAV, N.P.; PAL, A.; SHANKER, K. et al. Synergistic effect of silymarin and standardized extract of Phyllanthus amarus against $\mathrm{CCl}_{4-}$ induced hepatotoxicity in Rattus norvegicus. Phytomedicine, v.15, p.1053-1061, 2008.

YANG, H.L.; CHEN, S.C.; CHANG, N.W. et al. Protection from oxidative damage using Bidens pilosa extracts in normal human erythrocytes. Food Chem. Toxicol., v.44, p.1513-1521, 2006.

YUAN, L.P.; CHENA, F.H.; LING, L. et al. Protective effects of total flavonoids of Bidens pilosa $L$. (TFB) on animal liver injury and liver fibrosis. J. Ethnopharm., v.116, p.539-546, 2008. 